\title{
Use of Cloud Computing in Oil and Gas Industry
}

\section{Introduction}

Today "cloudy technologies" is the name of one of the main driving forces not only in the field of IT, but in allied industries as well. According to the standard definition "cloud computing" is the use of computing resources (hardware and software) delivered as a service over a network (typically the Internet). The name comes from the use of a cloud-shaped symbol as an abstraction for the complex infrastructure, that it contains, in system diagrams. Cloud computing entrusts remote services with a user's data, software and computation [4].

The software of processing of geographical spatial information in the majority of cases is rather expensive: not each company can afford it. In addition, there can be situations when application of such programs is necessary only from time to time. In both cases it is possible to pay for the service of access to a certain software for the concrete period. It will be much cheap that to buy it. Similar benefit can be got also by software developers who will be able to have access to necessary libraries, bases of spatial data and the services being in a cloud.

Users won't need anymore service of computing capacities demanded for functioning of software packages. From the companies providing cloudy services, the technology of the virtualization existing in cloud computing, allows to transform one server to a large number of virtual cars, maximizes possibilities of computing capacities [3], their use is more effective.

\section{Service Models}

Cloud computing providers offer their services according to several fundamental models: infrastructure as a service (IaaS), platform as a service (PaaS), and

* Taras Shevchenko National University of Kyiv, Geodesy and Cartography Department, Kyiv, Ukraine 
software as a service (SaaS), where IaaS is the most basic and each higher model abstracts from the details of the lower models (Fig. 1). Other key components in XaaS are described in a comprehensive taxonomy model published in 2009, such as Strategy-as-a-Service, Collaboration-as-a-Service, Business Process-as-a-Service, Database-as-a-Service, etc. In 2012, network as a service (NaaS) and communication as a service (CaaS) were officially included by ITU (International Telecommunication Union) as part of the basic cloud computing models, recognized service categories of a telecommunication-centric cloud ecosystem.

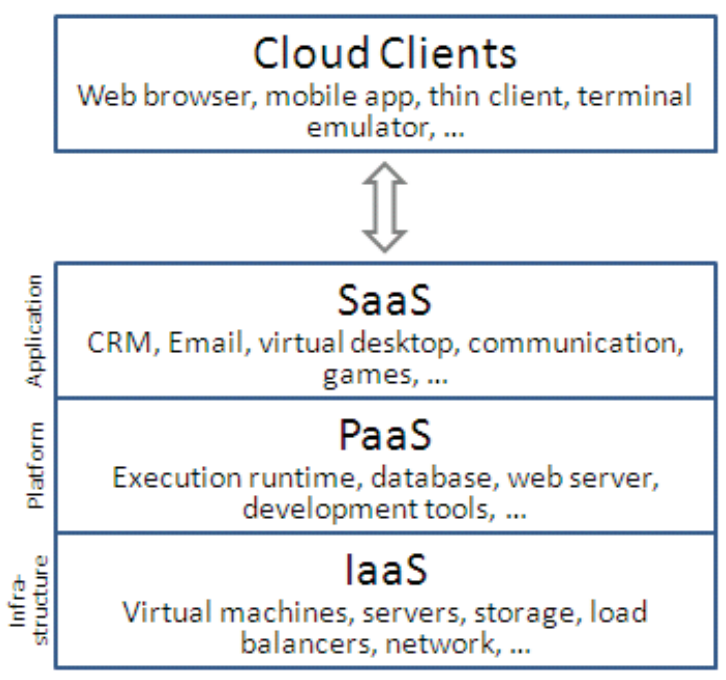

Fig. 1. Service model

\section{Infrastructure as a Service (IaaS)}

In the most basic cloud-service model, providers of IaaS offer computers physical or (more often) virtual machines - and other resources. (A hypervisor, such as Xen or KVM, runs the virtual machines as guests. Pools of hypervisors within the cloud operational support-system can support large numbers of virtual machines and the ability to scale services up and down according to customers' varying requirements.) IaaS clouds often offer additional resources such as a virtual-machine disk image library, raw (block) and file-based storage, firewalls, load balancers, IP addresses, virtual local area networks (VLANs), and software bundles. IaaS-cloud providers supply these resources on-demand from their large pools installed in data centers. For wide-area connectivity, customers can use either the Internet or carrier clouds (dedicated virtual private networks).

To deploy their applications, cloud users install operating-system images and their application software on the cloud infrastructure. In this model, the cloud user 
patches and maintains the operating systems and the application software. Cloud providers typically bill IaaS services on a utility computing basis: cost reflects the amount of resources allocated and consumed.

\section{Platform as a Service (PaaS)}

In the PaaS model, cloud providers deliver a computing platform typically including operating system, programming language execution environment, database, and web server. Application developers can develop and run their software solutions on a cloud platform without the cost and complexity of buying and managing the underlying hardware and software layers. With some PaaS offers, the underlying computer and storage resources scale automatically to match application demand such that cloud user does not have to allocate resources manually.

\section{Software as a Service (SaaS)}

In the SaaS model, cloud providers install and operate application software in the cloud and cloud users access the software from cloud clients. Cloud users do not manage the cloud infrastructure and platform where the application runs. This eliminates the need to install and run the application on the cloud user's own computers, which simplifies maintenance and support. Cloud applications are different from other applications in their scalability, which can be achieved by cloning tasks onto multiple virtual machines at run-time to meet changing work demand. Load balancers distribute the work over the set of virtual machines. This process is transparent to the cloud user, who sees only a single access point. To accommodate a large number of cloud users, cloud applications can be multitenant, that is, any machine serves more than one cloud user organization. It is common to refer to special types of cloud based application software with a similar naming convention: desktop as a service, business process as a service, test environment as a service, communication as a service

The pricing model for SaaS applications is typically a monthly or yearly flat fee per user, so price is scalable and adjustable if users are added or removed at any point.

\section{Network as a Service (NaaS)}

A category of cloud services where the capability provided to the cloud service user is to use network/transport connectivity services and/or inter-cloud network connectivity services. NaaS involves the optimization of resource allocations by considering network and computing resources as a unified whole.

Traditional NaaS services include flexible and extended VPN, and bandwidth on demand. NaaS concept materialization also includes the provision of a virtual network service by the owners of the network infrastructure to a third party (VNP $-\mathrm{VNO})$. 
Thus IDC forecasts public IT cloud services spending will achieve USD 100 billion in 2016, generating $41 \%$ of growth in five key IT categories [2]. Worldwide spending on public IT cloud services will be more than USD 40 billion in 2012 and is expected to approach USD 100 billion in 2016, according to a new forecast from International Data Corporation (IDC). Over the 2013-2016 forecast period, public IT cloud services will enjoy a compound annual growth rate (CAGR) of $26.4 \%$, five times that of the IT industry overall, as companies accelerate their shift to the cloud services model (Fig. 2) for IT consumption.

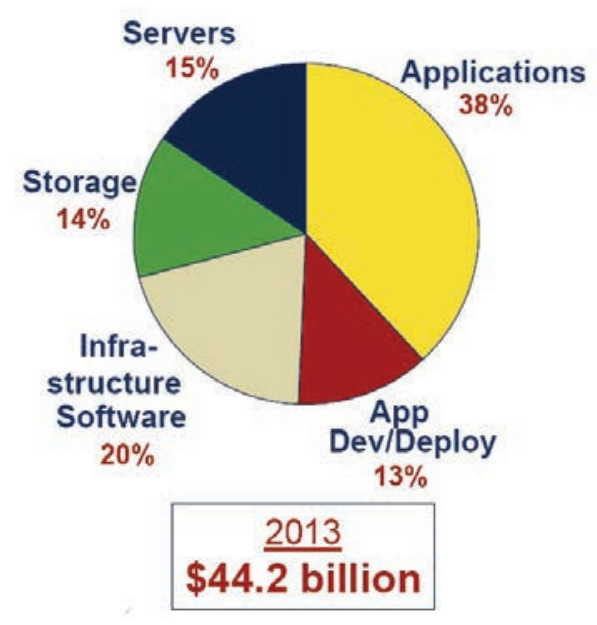

Fig. 2. Founds allocated for infrastructure

"The IT industry is in the midst of an important transformative period as companies invest in the technologies that will drive growth and innovation over the next two to three decades," said Frank Gens, senior vice president and chief analyst at IDC. "By the end of the decade, IDC expects at least $80 \%$ of the industry's growth, and enterprises' highest-value leverage of IT, will be driven by cloud services and the other $3^{\text {rd }}$ Platform technologies."[2].

By 2016, public IT cloud services will account for 16\% of IT revenue in five key technology categories: applications, system infrastructure software, platform as a service (PaaS), servers, and basic storage. More significantly, cloud services will generate $41 \%$ of all growth in these categories by 2016. "Quite simply, vendor failure in cloud services will mean stagnation," Gens added [2].

Software as a service (SaaS), which is the combination of applications as a service and system infrastructure software as a service, will claim the largest share of public IT cloud services spending over the next five years. But other categories, notably basic storage and platform as a service, will show faster growth. Accelerating PaaS rollouts over the next 12-18 months will be critical to maintaining strong cloud momentum. 
The oil and gas industry is knowledge intensive and is even experimenting with social networking. The question is whether the industry, given its global nature, need for security, and existing legacy infrastructure, will take to the cloud.

The public cloud is a deployment model where the cloud is open to a largely unrestricted universe of potential users. In fact, the public cloud has been a part of the IT landscape for several years, mainly in the form of software as a service. Public cloud includes but goes beyond software as a service to IT infrastructure or platform as a service. The private cloud is designed for restricted access to a single enterprise (or extended enterprise). A hybrid cloud combines a private cloud with the use of public cloud, especially when internal private cloud resources are strained.

The cloud services model, by leveraging all eight of these attributes together, makes business and consumer cloud services easier and cheaper and often better to consume than through traditional delivery modes. These attributes cost lower (for customers and suppliers), they provide better speed and simplification of access, speed and fine-tuning provisioning (in line with true demand/usage), greatly increasing the number and variety of available services (thanks to lower development and deployment costs and standards), and improving the potential to integrate.

Despite the popularity of the cloud in some applications, cloud computing has still not aggressively penetrated the oil and gas industry relative to the pace of adoption in other industries. According to IDC's 2010 Vertical Group Survey, 10.3\% of oil and gas companies are currently using or implementing cloud computing and $7 \%$ have cloud computing on their technology road map (Fig. 3). Forty-two percent of the companies that said they would use the cloud in 2010 were spending less than $5 \%$ of their IT budget on cloud.

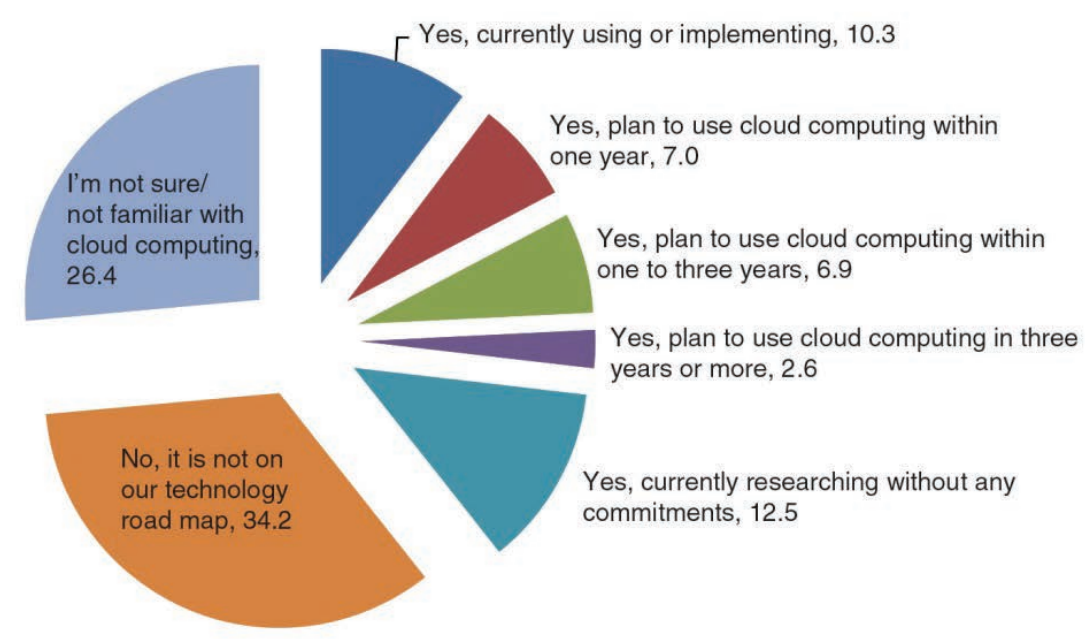

Fig. 3. Respondent's answers to the questions "Is cloud computing currently on your organization's technology roadmap?" 
There are several areas that are considered to be problematic. There is reluctance in the industry to have data stored outside of the firewall. There is a concern not only with intrusions that could compromise IT, but also with protection of trade secrets, especially when it comes to sensitive areas such as well logs. There is also an issue of scale for some applications. For example, much of exploration and production depends on 3D rendering and graphics accelerators, which haven't yet to make it off of the workstation because of the size of the files and speed required for viewing. Another consideration is the sunk investment in legacy IT applications and infrastructure that the industry has already made.

These barriers point to greater adoption by oil and gas of the private over the public cloud. In fact, many companies are already taking the first step to the private cloud through server and storage virtualization as means to make better use of system resources and lower the overall costs of operating the infrastructure. Oil and gas companies need environments with higher performance for large-scale applications. Virtualization permits workstations to run two separate operating systems concurrently, each running high-end, petro-technical applications at near native dedicated performance.

Virtualization does not equal the private cloud, however. The cloud adds a layer of orchestration to coordinate delivery across servers, storage and networks, and provides integrated provisioning. A private cloud becomes a pool of on-demand resources. Because of IT investment already made by many of the oil and gas majors, a private cloud model supports a higher level of flexibility, and more control (full access to all the bits and components) as opposed to a shared services model. It allows company IT and business personnel to come up the learning curve in a familiar environment.

Use of the private cloud has own challenges, however. It can take a lot of work to set up the right structures and identify the right tools to build and manage the private cloud. Interoperability is a challenge as well. There is also a question about how to charge internally for services provided by the private cloud, whereas public cloud charges are more straightforward.

As the cloud begins to show how it can address speed, cost, scale, and security, adoption of cloud use will likely increase in the industry, not just for private cloud, but for public cloud as well.

Already the oil and gas industry is using the public cloud for:

- Rapidly changing regulations. Because oil and gas is a global industry that is highly regulated, being able to depend on software as a service to manage the changes in regulations and reporting frees the companies from using personnel to track new developments, especially in the areas of health, safety, and environmental regulation.

- Capital project management involving many parties. Sharing specifications, drawings and other documents, while ensuring appropriate approvals, has always been labor intensive and subject to version confusion. 
The global oil and gas industry investment in public cloud (application software, servers, storage, application development and deployment software, and systems integration software) will rise from USD 1 billion in 2012 to more than USD 2 billion in 2014. North American spending will make up about half of that spending, given the faster adoption of the cloud in this region. Still to come is a sizing for the private cloud market.

In the field of high technology today there is a trend shift toward cloud computing. According to statistics, a search engine Google cloud is more famous then cluster and network [1].

Direction is developing dynamically and has opportunities for integration with existing geographic information tools.

Cartographic small companies can gain access to powerful and expensive package of spatial data processing, computing power and infrastructure, for an amount less than what they would have to spend to buy all the necessary equipment and software.

Major mapping companies that already have the necessary hardware and software, with the use of cloud technologies can provide a quick access to their geo-services.

On the scale of a national ISD (infrastructure of spatial data) implementation of cloud computing is while hindered by a number of problems common with the use of spatial data and technology associated with them.

The existing course on the innovative technologies one of which is cloud computing, needs to be supported. The delay in the solution of these problems can turn back today large expenses in the future.

\section{References}

[1] Buyya R., Yeo Chee Shin, Venugopal S., Broberg J., Brandic I.: Cloud Computing and Emerging IT Platforms Vision, Hype, and Reality for Delivering Computing as the 5th Utility. Future Generation Computer Systems, vol. 25, 2009, pp. 599-616, [on-line:] http://www.sciencedirect.com/science/article/ pii/S0167739X08001957\# [access: November 30, 2013].

[2] IDC - Press Release. Framingham, September 11, 2012, [on-line:]: http:// www.idc.com/getdoc.jsp?containerId=prUS23684912\#.USASCC9GqSo [access: November 30, 2013].

[3] Kouyoumjian V.: The New Age of Cloud Computing and GIS. ArcWatch: Your e-Magazine for GIS News, Views, and Insights. January, 2010, [on-line:] http://www.esri.com/news/arcwatch/0110/feature.html [access: November 30, 2013].

[4] Mell P., Grance T.: The NIST Definition of Cloud Computing, special publication 800-145, [on-line:] http://csrc.nist.gov/publications/nistpubs/800-145/ SP800-145.pdf [access: February 15, 2013]. 